\title{
Contribution of charm to rare radiative leptonic B-decays
}

\author{
Anastasiia Kozachuk ${ }^{1,2, *}$ \\ ${ }^{1}$ D. V. Skobeltsyn Institute of Nuclear Physics, M. V. Lomonosov Moscow State University, 119991, \\ Moscow, Russia \\ ${ }^{2}$ M. V. Lomonosov Moscow State University, Faculty of Physics, 119991, Moscow, Russia
}

Abstract. We revisit rare radiative leptonic decays $B_{d, s} \rightarrow \gamma \mu^{+} \mu^{-}$and $B_{d, s} \rightarrow \gamma e^{+} e^{-}$in the Standard Model and obtain predictions for a number of differential distributions in these decays with special emphasis on the contribution of charm-quark loops.

\section{Introduction}

Rare FCNC decays of B-mesons are forbidden at tree level in the Standard Model and occur only via loop diagrams, which makes the branching ratios of the decays very small, typical order is $10^{-8}-10^{-10}$ [1]. New unknown particles can contribute to the loops and hence such processes are expected to be sensitive to the potential effects of New Physics. Several decays of this type have been studied experimentally (see discussion in [2-5]). They are, for example, $B \rightarrow K^{+} \ell^{+} \ell^{-}$and $B \rightarrow K^{* 0} \ell^{+} \ell^{-}$decays, which have been studied at LHCb in order to test lepton flavor universality $[6,7]$. So far, a few deviations from the Standard Model of the order of $2-3 \sigma$ have been observed.

This talk presents the results of our recent work [8] where rare radiative leptonic decays $B_{d, s} \rightarrow \gamma \mu^{+} \mu^{-}$and $B_{d, s} \rightarrow \gamma e^{+} e^{-}$have been analyzed. Rare radiative leptonic $B$-decays also represent FCNC decays; however, their amplitudes contain a number of additional contributions compared to FCNC decays with a meson and $l^{+} l^{-}$pair in the final state.

\section{Effective Hamiltonian}

Only loop diagrams contribute to $B_{s, d} \rightarrow \gamma \ell^{+} \ell^{-}$decays. The penguin diagram which gives one of the main contributions is presented in Fig. 1 . All the three quarks $\mathrm{u}, \mathrm{c}$ and $\mathrm{t}$ are possible

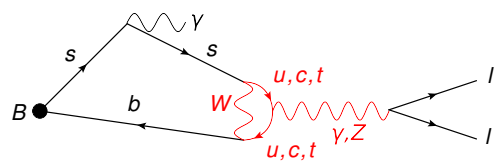

Figure 1. Penguin diagram contributing to $B_{s} \rightarrow \gamma \ell^{+} \ell^{-}$ decays

in the loop. Exchange of heavy virtual particles (W, Z bosons and t-quarks) can be reduced to effective quasilocal interactions. After heavy degrees of freedom are integrated out, one comes to the effective Hamiltonian - Wilson OPE (1), which describes dynamics at the scale

*e-mail: anastasiia.kozachuk@cern.ch 
$\mu$, appropriate for B-decays [16-18] [we use the sign convention for the effective Hamiltonian and the Wilson coefficients adopted in [19, 20]]:

$$
H_{\mathrm{eff}}^{b \rightarrow q}=\frac{G_{F}}{\sqrt{2}} V_{t q}^{*} V_{t b} \sum_{i} C_{i}(\mu) O_{i}^{b \rightarrow q}(\mu),
$$

where $G_{F}$ is the Fermi constant. This is a standard framework for describing rare FCNC $b \rightarrow q(q=s, d)$ transitions. The basis operators $O_{i}^{b \rightarrow q}(\mu)$ contain only light degrees of freedom ( $u, d, s, c$, and $b$-quarks, leptons, photons and gluons). The contributions of the heavy degrees of freedom of the SM ( $W, Z$ bosons, and $t$-quarks) are encoded in the Wilson coefficients $C_{i}(\mu)$. Light quarks (u and c) stay dynamical and require a different approach.

For the $\bar{B}_{s} \rightarrow \gamma l^{+} l^{-}$decays the following terms in (1) should be taken into account ${ }^{1}$ (the $\bar{B}_{d} \rightarrow \gamma l^{+} l^{-}$case is obtained with the obvious replacement $s \rightarrow d$ ):

$$
\begin{aligned}
H_{\mathrm{eff}}^{b \rightarrow} s^{+} l^{-} & =\frac{G_{F}}{\sqrt{2}} \frac{\alpha_{\mathrm{em}}}{2 \pi} V_{t b} V_{t s}^{*}\left[-2 i m_{b} \frac{C_{7 \gamma}(\mu)}{q^{2}} \cdot \bar{s} \sigma_{\mu \nu} q^{v}\left(1+\gamma_{5}\right) b \cdot \bar{l} \gamma^{\mu} l\right. \\
& \left.+C_{9 V}(\mu) \cdot \bar{s} \gamma_{\mu}\left(1-\gamma_{5}\right) b \cdot \bar{l} \gamma^{\mu} l+C_{10 A}(\mu) \cdot \bar{s} \gamma_{\mu}\left(1-\gamma_{5}\right) b \cdot \bar{l} \gamma^{\mu} \gamma_{5} l\right] .
\end{aligned}
$$

As already noted, the light degrees of freedom remain dynamical and their contributions should be taken into account separately. The relevant terms in $H_{\mathrm{eff}}^{b \rightarrow s}$ are those containing four-quark operators:

$$
H_{\mathrm{eff}}^{b \rightarrow s \bar{c} c}=-\frac{G_{F}}{\sqrt{2}} V_{c b} V_{c s}^{*}\left\{C_{1}(\mu) O_{1}+C_{2}(\mu) O_{2}\right\}
$$

with

$$
O_{1}=\bar{s}^{j} \gamma_{\mu}\left(1-\gamma_{5}\right) c^{i} \bar{c}^{i} \gamma^{\mu}\left(1-\gamma_{5}\right) b^{j}, \quad O_{2}=\bar{s}^{i} \gamma_{\mu}\left(1-\gamma_{5}\right) c^{i} \bar{c}^{j} \gamma^{\mu}\left(1-\gamma_{5}\right) b^{j},
$$

and the similar terms with $c \rightarrow u(i, j$ here are color indices).

\section{Contributions with t-quarks in the loops}

The diagrams generated by the t-quarks (as well as other heavy particles of the SM) in the loops are presented in Fig. 2 and Fig. 3. The $B$-decay amplitudes corresponding to these
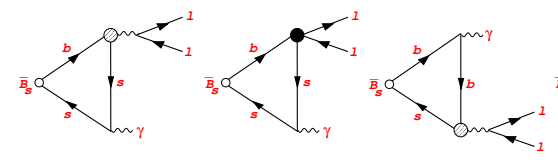

Figure 2. Diagrams, contributing to the $\bar{B}_{s} \rightarrow \gamma l^{+} l^{-}$decays. Effective vertices (black and dashed circles) correspond to loops with t-quarks and other heavy particles of the SM, which are integrated out

diagrams are described in terms of $B$-meson-to-photon transition form factors. The main problem here is the presence of the B-mesons in the initial state, which makes it necessary to take into account non-perturbative QCD effects. In our work [8] the form factors have been calculated in the framework of the relativistic quark model [9-13] that represents the form factors as relativistic spectral representations via the meson wave functions and allows one to obtain the form factors in the wide range of momentum transfer. The model contains a number of parameters (i.e. the constituent quark masses and the hadron wave functions) that

\footnotetext{
${ }^{1}$ Our notations and conventions are: $\gamma^{5}=i \gamma^{0} \gamma^{1} \gamma^{2} \gamma^{3}, \sigma_{\mu v}=\frac{i}{2}\left[\gamma_{\mu}, \gamma_{v}\right], \varepsilon^{0123}=-1, \epsilon_{a b c d} \equiv \epsilon_{\alpha \beta \mu \nu} a^{\alpha} b^{\beta} c^{\mu} d^{\nu}$, $e=\sqrt{4 \pi \alpha_{\mathrm{em}}}$.
} 
have been fixed by the requirement to reproduce weak decay constants and a few well-known form factors of weak decays of heavy mesons obtained from lattice, sum rules or experiment.

Fig. 4 shows another contribution to the amplitude of $B_{s, d} \rightarrow \gamma \ell^{+} \ell^{-}$decays, the bremsstrahlung contribution. The bremsstrahlung amplitude is given in terms of the B-meson decay constant $f_{B}[14,15]$ and is proportional to the mass of the lepton in the final state.
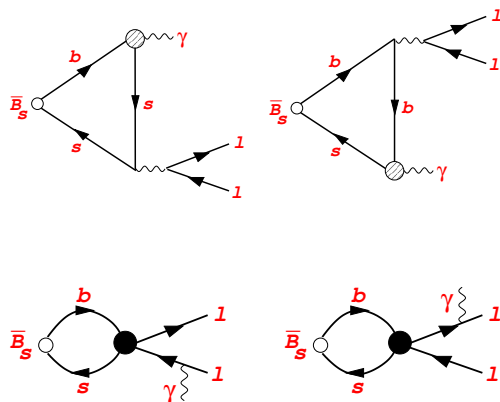

Figure 3. Diagrams, corresponding to the contribution when the real photon is emitted from the penguin FCNC vertex, whereas the virtual photon is emitted from the valence quarks of the B-meson

Figure 4. Diagrams, corresponding to the bremsstrahlung contribution

\section{Contributions of the charm-loops}

Whereas heavy degrees of freedom $(t, W, Z)$ have been integrated out and described by the effective Hamiltonian, light degrees of freedom, in particular $c$ and $u$ quarks, remain dynamical and their contributions in the loops should be taken into account separately. The

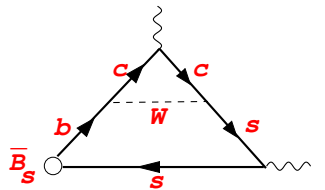

(a)

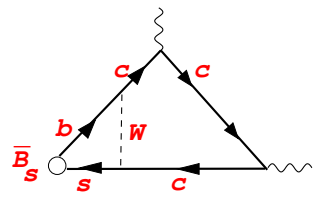

(b)
Figure 5. Diagrams with c-quarks in the loops: (a) - penguin diagram, (b) - weak annihilation diagram

diagrams corresponding to the contributions of the charm-loops are presented in Fig. 5: the penguin diagram (a) and the weak-annihilation diagram (b).

The contribution of the diagram of the weak annihilation type (b) is small in comparison to that of the penguin diagram and we do not discuss it here despite the fact that its contribution was taken into account. For the penguin diagram when the W-boson line is reduced to a point we obtain the c-quark loop for the full range of $q^{2}\left(q^{2}\right.$ is the squared momentum of the lepton pair). Hence, we have all the cc-resonances in the kinematical region of the process, which results in uncertainties in theoretical predictions.

The charm-loop contributions to the $B_{s} \rightarrow \gamma l^{+} l^{-}$amplitude are related to the following matrix element:

$$
\begin{aligned}
H_{\mu \alpha}\left(k^{\prime}, k\right)=- & \frac{G_{F}}{\sqrt{2}} V_{c b} V_{c s}^{*} e\left[\epsilon_{\mu \alpha k^{\prime} k} H_{V}-i\left(g_{\alpha \mu} k k^{\prime}-k_{\alpha}^{\prime} k_{\mu}\right) H_{A}\right. \\
& \left.-i\left(k_{\alpha}^{\prime}-\frac{k k^{\prime}}{k^{2}} k_{\alpha}\right)\left(k_{\mu}-\frac{k k^{\prime}}{k^{\prime 2}} k_{\mu}^{\prime}\right) H_{3}\right]
\end{aligned}
$$

The form of the matrix element is dictated by the conservation of the vector charm-quark and the electromagnetic currents, which requires $k^{\alpha} H_{\mu \alpha}\left(k^{\prime}, k\right)=0$ and $k^{\prime \mu} H_{\mu \alpha}\left(k^{\prime}, k\right)=0$ (notice the absence of any contact terms), with the invariant form factors $H_{i}$ depending on 
two variables, $H_{i}\left(k^{\prime 2}, k^{2}\right)$. The singularities in the projectors at $k^{2}=0$ and $k^{2}=0$ should not be the singularities of the amplitude $H_{\alpha \mu}\left(k, k^{\prime}\right)$, leading to the constraints

$$
H_{3}\left(k^{\prime 2}=0, k^{2}\right)=H_{3}\left(k^{\prime 2}, k^{2}=0\right)=0 .
$$

As the result, $H_{3}$ does not contribute to the amplitude of rare radiative leptonic $B$-decays.

For the charm-loop contributions given by $H_{i}\left(q^{2}, 0\right)$ it is necessary to describe a wide range $0<q^{2}<M_{B}^{2}$, which includes the region of the charmonium resonances. Perturbative QCD cannot be applied here and non-perturbative approaches based on hadron degrees of freedom are necessary, see discussion in [21-28]. For $H_{i}\left(q^{2}, 0\right)$ one may write dispersion representation in $q^{2}$ with two subtractions, similar to the $B \rightarrow K^{*} l^{+} l^{-}$amplitudes $H_{1,2}$ of [21]:

$$
H_{i}\left(q^{2}, 0\right)=a_{i}+b_{i} q^{2}+\left(q^{2}\right)^{2}\left\{\sum_{\psi=J / \psi, \psi^{\prime}} \frac{f_{\psi} \mathcal{A}_{B \psi \gamma}^{i}}{m_{\psi}^{3}\left(m_{\psi}^{2}-q^{2}-i m_{\psi} \Gamma_{\psi}\right)}+h_{i}\left(q^{2}\right)\right\}, \quad i=V, A,
$$

where $a_{i}$ and $b_{i}$ are the (unknown) subtraction constants and the functions $h_{i}\left(q^{2}\right)$ describe the hadron continuum including the broad charmonium states lying above the $D D$ threshold. At $q^{2}>4 M_{D}^{2}$, we take into account the contributions of the known broad vector $\psi_{n}(n=3, \ldots, 6)$ resonances (and add a heavier effective pole) to $h\left(q^{2}\right)$ in (7), for the detailed formula see [8].

The numerical values of the coefficients $a_{i}$ and $b_{i}$ are obtained by matching (7) to the results of light-cone sum rules [21] at small $q^{2}$; for details of this procedure see [8]. A discussion of subtleties in the applications of light-cone sum rules to FCNC decays we refer to our recent paper [29].

The absolute values of the amplitudes $\mathcal{A}_{B \psi \gamma}^{i}$ may be determined from the experimental data. It should be understood, however, that nonfactorizable gluons may generate complex relative phases between the contributions of different charmonia [30]. One of the possible consequences of nonfactorizable gluon exchanges might be e.g. different relative signs of the charmonia contributions to the $B \rightarrow \gamma l^{+} l^{-}$amplitude [21]. These possible phases which cannot be determined by pQCD-based calculations is one of the main sources of the theoretical uncertainties for rare radiative leptonic decays.

\section{Results}

The differential branching ratios are shown in Fig. 6. The results in Fig. 6 correspond to the description of the charm-loop effects according to Eq. (7) and adding the contributions of the broad charmonia, and further assuming that all charmonia contribute with the same positive sign (coinciding with the sign of the factorizable contribution). The subtraction constants $a$ and $b$ in Eq. (7) are determined by the requirement to reproduce the known results at $q^{2} \leq 4 m_{c}^{2}$, including non-factorizable corrections calculated in [21]. In the region $q^{2} \leq 6$ $\mathrm{GeV}^{2}$, the charming loops provide a mild contribution at the level of a few percent, and therefore the branching fractions in this region may be predicted with a controlled accuracy, mainly limited by the form-factor uncertainty. Our estimates read

$$
\begin{aligned}
& \left.\mathcal{B}\left(\bar{B}_{s} \rightarrow \gamma l^{+} l^{-}\right)\right|_{q^{2} \in[1,6] \mathrm{GeV}^{2}}=(6.01 \pm 0.08) 10^{-9} \\
& \left.\mathcal{B}\left(\bar{B}_{d} \rightarrow \gamma l^{+} l^{-}\right)\right|_{q^{2} \in[1,6] \mathrm{GeV}^{2}}=(1.02 \pm 0.15) 10^{-11} .
\end{aligned}
$$

The uncertainties in these estimates reflect merely the $10 \%$ uncertainty in the $B \rightarrow \gamma$ transition form factors. We would like to emphasize that in the case of the $B_{s} \rightarrow \gamma l^{+} l^{-}$transition, the dominant contribution is given by the narrow $\phi$-meson pole. The parameters of this pole are known quite well, leading to the small total uncertainty in the $B_{s} \rightarrow \gamma l^{+} l^{-}$decay rate 

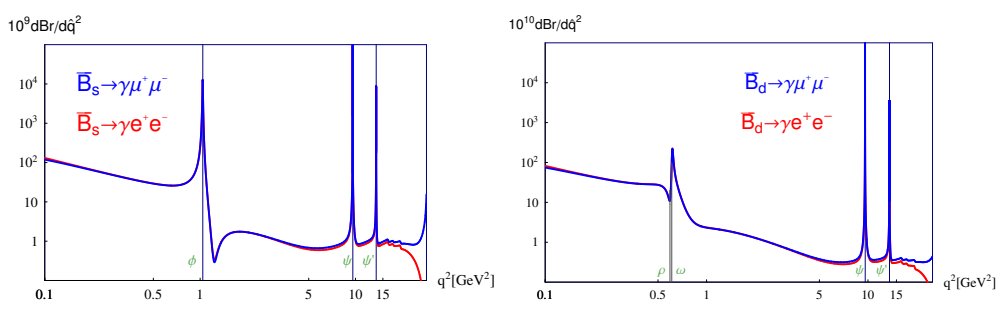

Figure 6. Differential branching fractions for $B_{s} \rightarrow \gamma l^{+} l^{-}$(left) and $B_{d} \rightarrow \gamma l^{+} l^{-}$(right) decays. Blue lines $-\mu^{+} \mu^{-}$final state, red lines $-e^{+} e^{-}$final state.

integrated over the range of $q^{2}=[1,6] \mathrm{GeV}^{2}$. In the case of the $B_{d} \rightarrow \gamma l^{+} l^{-}$transition, the known contribution of the vector resonances is less important, and as the result, the branching ratio uncertainty reflects to a large extent the form factor uncertainty of $10 \%$.

Fig. 7 shows the ratio $R_{\mu / e}$ of the differential distributions $\bar{B} \rightarrow \gamma \mu^{+} \mu^{-}$to $\bar{B} \rightarrow \gamma e^{+} e^{-}$. Such ratio is a standard observable for probing violations of the lepton universality in rare FCNC semileptonic decays $B \rightarrow(P, V) l^{+} l^{-}$; recently, this ratio was proposed as a useful variable also in radiative leptonic decays [31]. The ratio is found to be close to unity in the region $q^{2} \leq 5 \mathrm{GeV}^{2}$. Notice however, that in $B \rightarrow \gamma l^{+} l^{-}$decays, unlike the $B \rightarrow(P, V) l^{+} l^{-}$processes, the lepton-mass effects, mainly the bremsstrahlung contribution to the amplitude, come into the game, thus driving the ratio far above unity at $q^{2}>15 \mathrm{GeV}^{2}$. Here the interference between the bremsstrahlung contribution proportional to $m_{l}$ and the form factor contribution dominates the ratio. Thus, if the lepton universality is verified at small $q^{2}$, measuring $R_{\mu / e}$ in the region $q^{2}>15 \mathrm{GeV}^{2}$ provides a direct test of the $q^{2}$-dependence of the $B \rightarrow \gamma$ transition form factors.
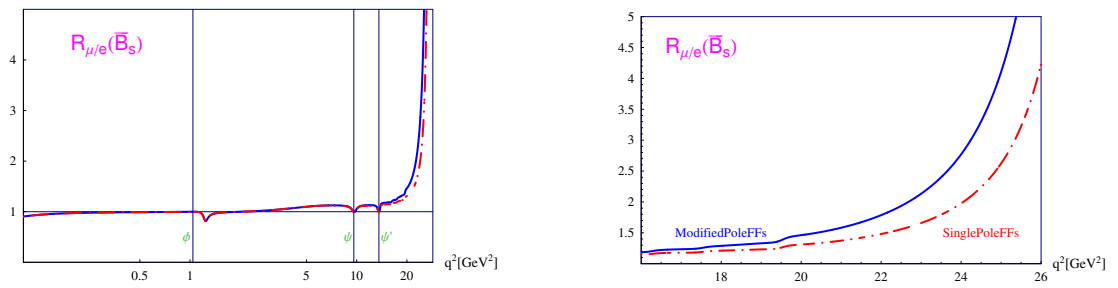

Figure 7. $R_{\mu / e}$ ratio for $B_{s} \rightarrow \ell^{+} \ell^{-} \gamma$ decays for different types of form factor parameterization: solid line for modified pole parameterization and dash-dotted line for single pole parameterization

The results for the forward-backward asymmetry in the $\bar{B}_{s} \rightarrow \gamma \mu \mu$ decay are shown in Fig. 8, for $\bar{B}_{d} \rightarrow \gamma \mu \mu$ the asymmetry has similar structure. The asymmetries are practically insensitive to the uncertainties in the $B \rightarrow \gamma$ transition form factors, as these uncertainties to large extent cancel each other in the asymmetries.

For more results and details see our work [8]. 

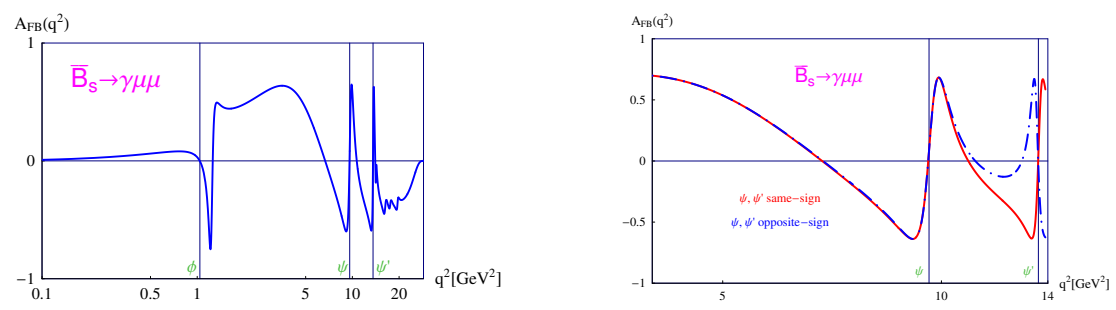

Figure 8. Forward-backward asymmetry for $\bar{B}_{s} \rightarrow \gamma \mu^{+} \mu^{-}$decay. The right plot shows the asymmetry at $q^{2}<M_{\psi^{\prime}}^{2}$ for two different relatives signs of $\psi$ and $\psi^{\prime}$ contributions

\section{Conclusions}

We performed a detailed theoretical analysis of rare radiative leptonic decays $B_{s, d} \rightarrow \mu^{+} \mu^{-} \gamma$ and $B_{s, d} \rightarrow e^{+} e^{-} \gamma$. We list a few of the results below:

1. The invariant form factors, which parameterize the amplitudes of the processes, were calculated in the wide range of momentum transfer within the relativistic quark model. The parameters of the model, such as constituent quark masses and parameters of the hadron wave functions were fixed by the requirement to reproduce weak decays constants and form factors obtained from lattice, sum rules or experiment. We obtained constraints for these form factors imposed by electromagnetic gauge invariance.

2. We performed a detailed study of the charm-loop contributions. We demonstrated that the results for the nonfactorizable corrections available at $q^{2}$ below the charm threshold do not allow one to resolve the possible ambiguity in the relative charmonium resonance phases. Additional inputs are necessary to determine the phases unambiguously.

3. We obtained differential distributions for the observables, namely the differential branching ratios, the forward-backward asymmetries and the $R_{\mu / e}\left(q^{2}\right)$, the ratio of the $B \rightarrow \gamma \mu^{+} \mu^{-}$over $B \rightarrow \gamma e^{+} e^{-}$differential distributions. We demonstrated, that the latter at large $q^{2}$ provides direct access to measuring the $q^{2}$-dependencies of the $B \rightarrow \gamma$ transition form factors, once the lepton universality is established from the data at low $q^{2}$

4. We presented numerical predictions for the branching ratios in the low region of $q^{2}$.

For more details see our main work [8].

\section{Acknowledgments}

The author is thankful to Dmitri Melikhov and Nikolai Nikitin for useful discussions. The work was done with the support of grant RNF 16-12-10280 of the Russian Science Foundation.

\section{References}

[1] A. Ali, [hep-ph/9606324].

[2] S. L. Glashow, D. Guadagnoli and K. Lane, Phys. Rev. Lett. 114, 091801 (2015) [arXiv:1411.0565].

[3] D. Guadagnoli and K. Lane, Phys. Lett. B 751, 54 (2015) [arXiv:1507.01412]. 
[4] D. Guadagnoli, D. Melikhov and M. Reboud, Phys. Lett. B 760, 442 (2016) [arXiv:1605.05718].

[5] D. Guadagnoli, Mod. Phys. Lett. A32, 1730006 (2017) [arXiv:1703.02804].

[6] LHCb Collaboration, R. Aaij et al., Phys. Rev. Lett. 113, 151601 (2014) [arXiv:1406.6482]

[7] LHCb Collaboration, R. Aaij et al., JHEP 1708, 055 (2017) [arXiv:1705.05802].

[8] A. Kozachuk, D. Melikhov and N. Nikitin, Phys. Rev. D 97, no. 5, 053007 (2018) [arXiv:1712.07926 [hep-ph]]

[9] D. Melikhov, Phys. Rev. D 53, 2460 (1996) [hep-ph/9509268].

[10] D. Melikhov, Phys. Rev. D 56, 7089 (1997) [hep-ph/9706417].

[11] D. Melikhov, Eur. Phys. J. direct 4, 1 (2002) [hep-ph/0110087].

[12] M. Beyer and D. Melikhov, Phys. Lett. B 436, 344 (1999) [hep-ph/9807223].

[13] D. Melikhov and B. Stech, Phys. Rev. D 62, 014006 (2000) [hep-ph/0001113].

[14] F. Kruger and D. Melikhov, Phys. Rev. D 67, 034002 (2003) [hep-ph/0208256].

[15] D. Melikhov and N. Nikitin, Phys. Rev. D 70, 114028 (2004) [hep-ph/0410146].

[16] B. Grinstein, M. J. Savage and M. B. Wise, Nucl. Phys. B 319271 (1989).

[17] A. J. Buras and M. Munz, Phys. Rev. D 52, 186 (1995) [hep-ph/9501281].

[18] G. Buchalla, A. J. Buras, and M. E. Lautenbacher, Rev. Mod. Phys. 68, 1125 (1996) [hep-ph/9512380].

[19] D. Melikhov, N. Nikitin and S. Simula, Phys. Lett. B 430, 332 (1998) [hep-ph/9803343].

[20] D. Melikhov, N. Nikitin and S. Simula, Phys. Rev. D 57, 6814 (1998) [hep-ph/9711362].

[21] A. Khodjamirian, T. Mannel, A. Pivovarov, and Y.-M. Wang, JHEP 09, 089 (2010) [arXiv:1006.4945].

[22] A. Ali, T. Mannel, T. Morozumi, Phys. Lett. B 273, 505 (1991).

[23] M. Beneke, G. Buchalla, M. Neubert, and C. T. Sachrajda, Eur. Phys. J. C61, 439 (2009) [arXiv:0902.4446].

[24] M. Ciuchini, M. Fedele, E. Franco, S. Mishima, A. Paul, L. Silvestrini, and M. Valli, JHEP 1606, 116 (2016) [arXiv:1512.07157].

[25] J. Lyon and R. Zwicky, [arXiv:1406.0566].

[26] S. Brass, G. Hiller, and I. Nisandzic, Eur. Phys. J. C77, 16 (2017) [arXiv:1606.00775].

[27] LHCb Collaboration, R. Aaij et al., Eur. Phys. J. C77, 161 (2017) [arXiv:1612.06764].

[28] T. Blake, U. Egede, P. Owen, G. Pomery, K. Petridis, [arXiv:1709.03921].

[29] A. Kozachuk and D. Melikhov, arXiv:1805.05720 [hep-ph].

[30] J. Lyon and R. Zwicky, arXiv:1406.0566 [hep-ph].

[31] D. Guadagnoli, M. Reboud, and R. Zwicky, JHEP 1711, 184 (2017) [arXiv:1708.02649]. 